\title{
Comparing SU(2) to SU(3) gluodynamics on large lattices
}

\author{
A. Sternbeck, L. von Smekal, D. B. Leinweber and A. G. Williams* \\ Centre for the Subatomic Structure of Matter (CSSM), Department of Physics, \\ University of Adelaide, SA 5005, Australia \\ Email: andre.sternbeck@adelaide.edu.au, lorenz.smekal@adelaide.edu.au, \\ dleinweb@physics.adelaide.edu.au, anthony.williams@adelaide.edu.au
}

We study the SU(2) gluon and ghost propagators in Landau gauge on lattices up to a size of $112^{4}$. A comparison with the SU(3) case is made and finite-volume effects are then investigated. We find that for a large range of momenta the SU(2) and SU(3) propagators are remarkably alike. In the low-momentum region we compare with recent results obtained in DSE studies on a 4-torus.

The XXV International Symposium on Lattice Field Theory

July 30 - 4 August 2007

Regensburg, Germany

\footnotetext{
*Speaker.
} 


\section{Introduction}

For quite some time now the infrared behaviour of the fundamental QCD Green's functions has been a focus of intense study (see, e.g., the reviews $[1,2]$ and references therein). Nonperturbative effects have been and continue to be investigated in parallel with various functional continuum methods and in lattice simulations. The infrared behaviour of gluon and quark propagators and vertex functions, especially in Landau gauge, thereby is of particular relevance for an understanding of confinement in the covariant formulation of QCD in terms of local quark and gluon fields [1]. They also serve for model building in a hadron phenomenology based on these elementary QCD correlations via Dyson-Schwinger and Bethe-Salpeter/relativistic Faddeev equations [1, 3, 4].

Looking back at an intensive decade of research, results obtained in studies of Dyson-Schwinger equations (DSEs) [5, 6], within stochastic quantisation [7, 8], and of the functional renormalisation group equations (FRGEs) $[9,10]$ all agree in an infrared vanishing gluon propagator $D$ coupled to an enhanced infrared-diverging ghost propagator $G$. In Landau gauge these are

$$
D_{\mu \nu}^{a b}\left(q^{2}\right)=\delta^{a b}\left(\delta_{\mu v}-\frac{q_{\mu} q_{v}}{q^{2}}\right) \frac{Z\left(q^{2}\right)}{q^{2}} \quad \text { and } \quad G^{a b}\left(q^{2}\right)=\delta^{a b} \frac{J\left(q^{2}\right)}{q^{2}},
$$

where the dressing functions $Z$ and $J$ are predicted to follow power laws, namely

$$
Z\left(q^{2}\right) \propto\left(q^{2}\right)^{\kappa_{D}} \quad \text { and } \quad J\left(q^{2}\right) \propto\left(q^{2}\right)^{-\kappa_{G}} \quad \text { for } \quad q^{2} \rightarrow 0
$$

with exponents satisfying $\kappa_{D}=2 \kappa_{G}$ [5]. This infrared behaviour which is determined by a single exponent $\kappa \equiv \kappa_{G}$ can be generalised to vertex functions with an arbitrary number of ghost and gluon legs in a simple counting scheme for the pure gauge theory [11]. Moreover, comparing DSEs and FRGEs the uniqueness of this behaviour has been shown in Ref. [12]. Under the additional assumption that the ghost-gluon vertex is finite and regular in the infrared, the value of the infrared exponent is given by $\kappa \approx 0.596[6,7,9]$.

Despite intensive efforts, however, this behaviour has so far not been confirmed in simulations of lattice Landau-gauge QCD in 4 dimensions (see, e.g., $[13,14]$ ). Rather the results of present lattice simulations are, more or less, in favour of a non-vanishing gluon propagator and a diverging ghost propagator with a $\kappa_{G}$ value much less than that given above. The remaining discrepancy between the functional approaches and lattice QCD results is quite unsatisfying and needs clarification.

One attempt at a better understanding of the discrepancy has been undertaken in DSE studies on a finite torus. For recent results see Refs. [15, 16]. There, qualitatively good agreement has been found when comparing the momentum dependence of gluon and ghost propagators as found in lattice QCD to the solutions from finite-volume DSEs (see, e.g., Fig. 8 in Ref. [15]). In addition, these finite-volume solutions approach the infinite-volume DSE results when increasing the volume. Therefore, the discrepancy between the functional approaches and lattice QCD results might be due to finite-volume effects. Also, recent lattice results for an SU(2) gauge theory in 2 dimensions [17] (on much larger lattices) are in quite compelling agreement with the corresponding infrared behaviour as predicted by the continuum studies.

The objective of this study is to provide more information about the infrared behaviour of the Landau-gauge gluon and ghost propagators from a lattice-QCD perspective. For this, we first 
Table 1: Number of configurations used for the different $\beta$ values and lattice sizes. Approximate values for the edge length $L$ are also given. For this we set $\sqrt{\sigma}=440 \mathrm{MeV}$ and use the $\sigma a^{2}$ results from Ref. [19].

\begin{tabular}{crrc|cccc}
\hline \hline$\beta$ & latt. & $L[\mathrm{fm}]$ & \#conf. & $\beta$ & latt. & $L[\mathrm{fm}]$ & \#conf. \\
\hline 2.3 & $16^{4}$ & 2.7 & 100 & 2.5 & $32^{4}$ & 2.8 & 100 \\
2.3 & $32^{4}$ & 5.5 & 100 & 2.5 & $48^{4}$ & 4.3 & 50 \\
2.3 & $56^{4}$ & 9.6 & 70 & 2.5 & $56^{4}$ & 5.0 & 70 \\
2.3 & $80^{4}$ & 13.7 & 67 & 2.5 & $80^{4}$ & 7.1 & 23 \\
2.3 & $112^{4}$ & 19.2 & 34 & 2.6 & $48^{4}$ & 3.1 & 100 \\
\hline \hline
\end{tabular}

present numerical evidence that towards the low-momentum region both propagators are independent of whether considering SU(2) or SU(3). Given this, we then use the numerically cheaper gauge group SU(2) to dig even deeper into the infrared region of lattice Landau-gauge QCD by using large symmetric lattice volumes. First results of this study have been presented previously [18].

For our study we use the standard Wilson gauge action to generate $\mathrm{SU}(2)$ gauge configurations for a couple of lattice sizes ranging from $16^{4}$ up to $112^{4}$ at $\beta=2.3,2.5$ and 2.6 (see Table 1 for details). After every 2000 hybrid-overrelaxation updates, the gauge configurations are gauge-fixed to Landau gauge using an overrelaxation algorithm. As stopping criterion we chose

$$
\max _{x} \operatorname{Tr}\left[\left(\nabla_{\mu} A_{x, \mu}\right)\left(\nabla_{\mu} A_{x, \mu}\right)^{\dagger}\right]<10^{-14} .
$$

$A_{x, \mu}$ are the lattice gluon fields given here in terms of gauge-fixed links $U_{x, \mu}$ as

$$
A_{x, \mu} \equiv A_{\mu}(x+\hat{\mu} / 2)=\left.\frac{1}{2 a i g_{0}}\left(U_{x, \mu}-U_{x, \mu}\right)\right|_{\text {traceless }}
$$

On each such gauge-fixed configuration the momentum-space gluon and ghost propagators are measured. On the lattice, the former is defined as the Monte-Carlo average of the correlator

$$
D_{\mu \nu}^{a b}=\left\langle\tilde{A}_{\mu}^{a}(k) \tilde{A}_{v}^{b}(-k)\right\rangle_{U}
$$

of Fourier-transformed gluon fields $\tilde{A}_{\mu}=\tilde{A}_{\mu}^{a} T^{a}$. The ghost propagator can be estimated by

$$
G^{a b}(k)=\frac{1}{V}\left\langle\sum_{x y}\left(M^{-1}\right)_{x y}^{a b} e^{i k(x-y)}\right\rangle_{U}
$$

where $M$ is the lattice Faddeev-Popov operator in Landau gauge. For a definition of $M$ and details on its inversion we refer to $[20,21]$ and references therein.

\section{Comparing $S U(2)$ to $S U(3)$ results}

Our results for the SU(2) gluon dressing function are shown in Fig. 1 (left) together with corresponding SU(3) data taken from Refs. [14, 22]. Looking at those figures, the data for the two gauge groups are remarkably alike almost for the whole momentum region. A small deviation, however, is observed around the hump which becomes more pronounced upon increasing the volume. 

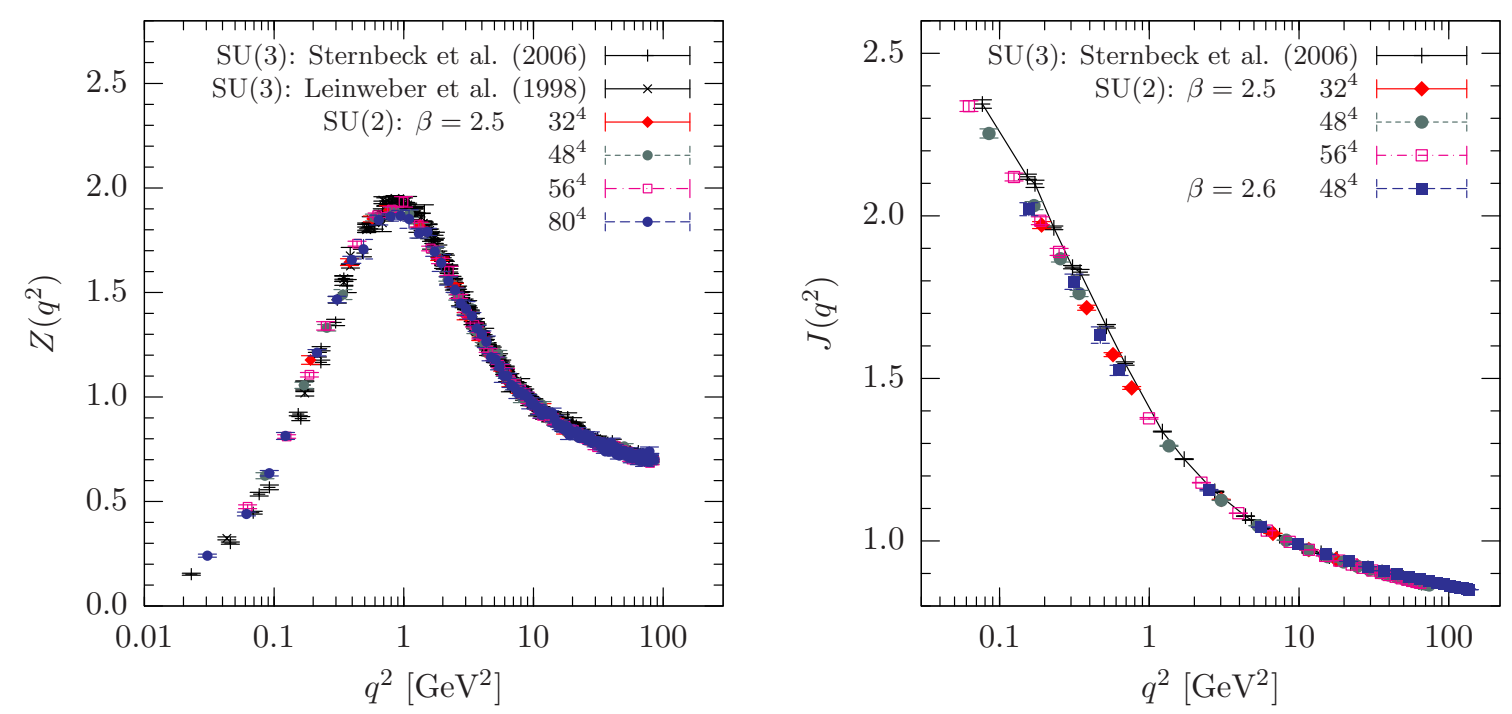

Figure 1: The gluon (left) and ghost (right) dressing functions vs. $q^{2}$ for the gauge groups SU(2) (colour symbols) and SU(3) (black symbols). All data sets have been renormalised at $\mu=3 \mathrm{GeV}$. The SU(3) ghost data are taken from Ref. [14].

The near $N_{c}$-independence becomes even more striking when comparing unrenormalised data at approximately equal lattice spacings and volumes for both gauge groups. Such a comparison is shown in Fig. 2 where we compare our $\mathrm{SU}(2)$ data for the gluon dressing function at $\beta=2.5$ and with $\mathrm{SU}(3)$ data at $\beta=6.0$ [14]. Obviously, the dependence on $N_{c}$ is rather small for a large range of momenta, but it seems not to be that small around $q=1 \mathrm{GeV}$. Also, at higher momenta a small $N_{c}$-dependence is visible as expected from perturbation theory given the $N_{c}$-dependence of the gluon dressing function beyond 1-loop.

Considering next the ghost fields, the renormalised ghost dressing functions of both gauge groups are compared in Fig. 1 (right). As for the gluon propagator, the dependence on the gauge group is rather small. This is also the case for the unrenormalised data (not

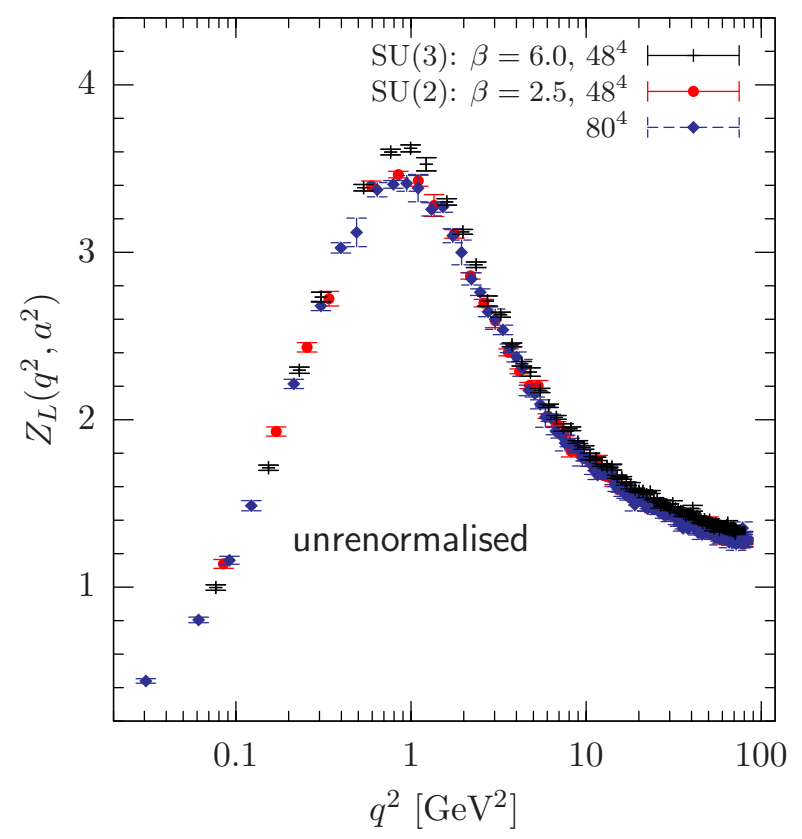

Figure 2: The unrenormalized gluon dressing function as a function of $q^{2}$ (in physical units) for the gauge group $\mathrm{SU}(2)$ and $\mathrm{SU}(3)$. shown). A slight deviation in the slope is visible towards low-lying momenta, however. Whether this is a gauge-group rather than a Gribov-copy issue remains to be investigated. Note that the Gribov ambiguity is expected to introduce some systematic error, in particular for the ghost propagator at lower momenta. For example, a bias towards larger values was observed in Ref. [20] for momenta $q<1 \mathrm{GeV}$. Since the Landau-gauge condition for $\mathrm{SU}(2)$ is less ambiguous than for 
$\mathrm{SU}(3)$ the slight deviation in the slope could be related to this rather than to $N_{c}$. We leave a detailed investigation of this for a future study.

\section{Studying finite-volume effects}

Given the rather small $N_{c}$-dependence, a finite-volume study has been performed for the numerically cheaper gauge group, $\mathrm{SU}(2)$. For this the coupling constant has been fixed to $\beta=2.3$. Various lattice sizes, ranging from $16^{4}$ to $112^{4}$, are considered. The lattice sizes in physical units are listed in Table 1. Our data for the gluon propagator is shown in Fig. 3 where data sets have neither been renormalised nor cone cut. ${ }^{1}$ The statistics for the $112^{4}$ lattice is still rather limited.

From the figure we conclude that the finite volume mainly affects the data only at the lowest (non-zero) lattice momentum. At larger momenta the data agrees quite well, within errors, comparing different lattice sizes. This suggests that indeed a cone cut does well in reducing finite-volume effects, but data at momenta like $k=(1,1,1,0)$ (or permutations thereof) need not be excluded necessarily from the analysis.

Comparing to the DSE studies on a torus [15], the finite-volume effect seen here agrees qualitatively with what has been observed there. However, at the presently available volumes we cannot confirm an infrared-decreasing gluon propagator. Note that according to Ref. [15] these volumes are already in a range where the gluon propagator is expected to decrease towards lower momenta. Rather, our data suggests that the gluon propagator stays finite in the zero-momentum limit. Similar results have been presented at this conference by Cucchieri et al. [24]. See also [25].

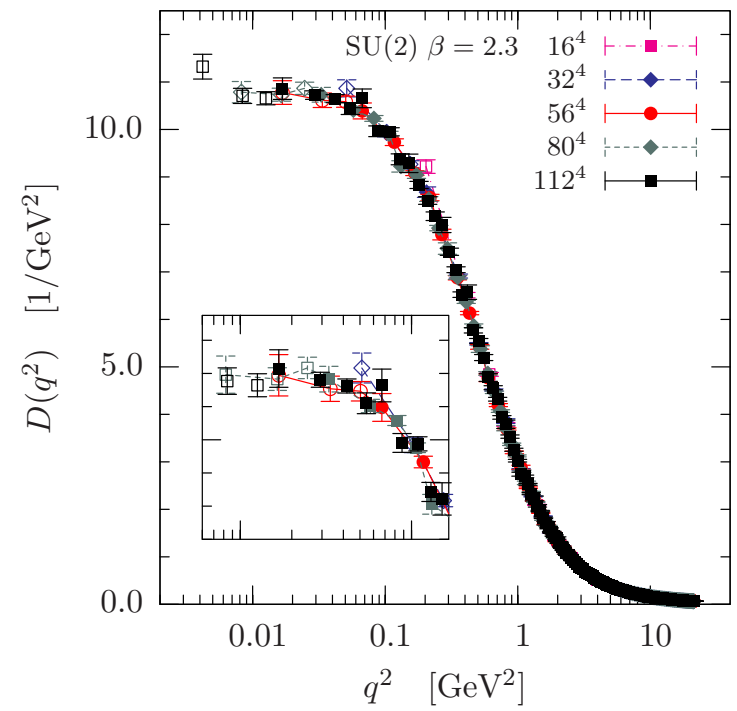

Figure 3: The unrenormalised SU(2) gluon propagator vs. $q^{2}$ calculated using different lattice sizes at $\beta=2.3$. Data shown with open symbols would be subject to a cone cut.

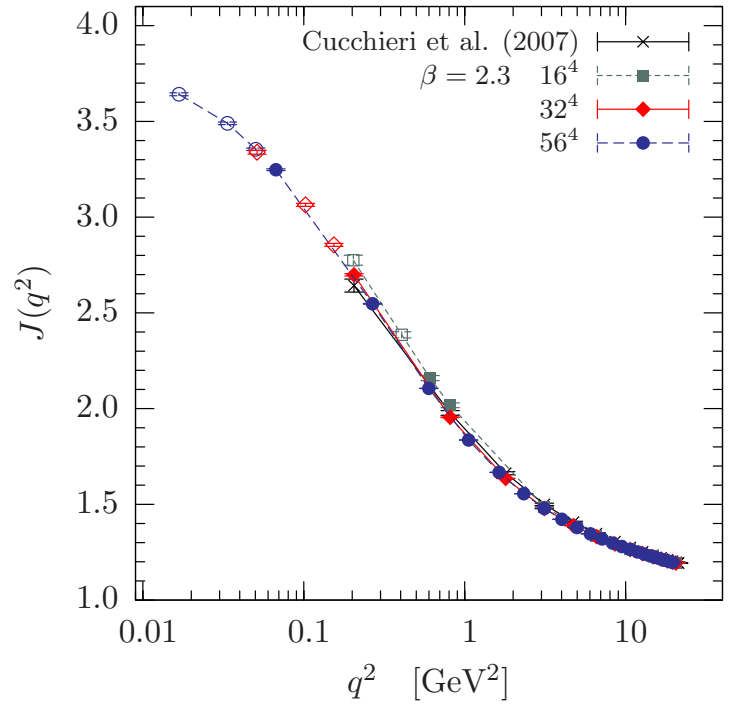

Figure 4: As in the left figure but for the SU(2) ghost dressing function. For comparison we include data from [23].

${ }^{1}$ A cone cut [22] is usually imposed to reduce finite-volume effects. Such data is specially flagged with open symbols here and must be interpreted with due care. This should not be confused with a cylinder cut which we do apply to reduce discretisation errors. 
The data for the $\mathrm{SU}(2)$ ghost dressing function for different volumes is shown in Fig. 4. There, a small volume $\left(16^{4}\right)$ biases the smaller-momentum data towards larger values. However, the dominant effect in the finite-volume DSE studies is in the opposite direction. It will be interesting to consider larger volumes to see if this effect persists. Calculations for the ghost propagator on a $80^{4}$ lattice are currently in progress and will be reported elsewhere.

\section{Conclusions}

We have calculated SU(2) gluon and ghost propagators in Landau gauge at different lattice spacings and volumes. A comparison of our data with corresponding $\mathrm{SU}(3)$ results reveals that there is very little $N_{c}$-dependence in the propagators over the whole momentum range as first reported in [18]. In particular, towards lower momenta, any signs for an $N_{c}$-dependence in the gluon propagator disappear. Similar findings have been presented in Refs. [26]. Moreover, the results found here are consistent with an approximate overall $1 / N_{c}$-scaling of the nonperturbative running coupling constant [5]

$$
\alpha_{s}\left(p^{2}\right)=\frac{g_{0}^{2}(a)}{4 \pi} Z_{L}\left(p^{2}, a^{2}\right) J_{L}^{2}\left(p^{2}, a^{2}\right) .
$$

Here $Z_{L}$ and $J_{L}$ are the lattice gluon and ghost dressing functions, respectively. $g_{0}^{2}(a)$ is the coupling constant at the cutoff scale $1 / a .^{2}$

Some small $N_{c}$-dependence is apparent in the gluon dressing function around its hump and at large momenta. A slight variation in the ghost dressing function towards lower momenta may or may not be related to $N_{c}$. Alternatively, this might be caused by the Gribov ambiguity which is known to introduce a systematic uncertainty here [20].

Our finite-volume study shows that the finite-lattice extent affects the gluon propagator at the lowest non-zero lattice momentum beyond the statistical error. The finite-volume effects qualitatively follow those found in DSE studies on a torus $[15,16]$. Of course, the volumes used at present, especially for the ghost propagator, may not yet be large enough to confirm the predicted finite-volume effects. However, our data provides indication of a plateau in the gluon propagator for momenta around and below approximately $200 \mathrm{MeV}$, or roughly $\Lambda_{\mathrm{QCD}}$, in contrast to the DSE results on a torus. To see the onset of an infrared suppression at even smaller momenta still requires a somewhat optimistic interpretation. Similar findings for $S U(3)$ have been presented at this conference [28]. It will be interesting to see whether and how the results change, in particular at low momenta, when using the slightly more expensive modified lattice Landau gauge of [29] in comparably large volumes.

\section{Acknowledgements}

This research was supported by the Australian Research Council. Generous grants of time on the Hydra, Aquila and Corvus Supercomputers, offered by the South Australian Partnership for Advanced Computing (SAPAC), allowed us to run simulations on such large lattice sizes. Grants of time on the APAC facility for parts of the project are also acknowledged.

\footnotetext{
${ }^{2}$ Note that recently a lattice study has been commenced which considers this definition of $\alpha_{s}$ to estimate the $\Lambda$ parameter of QCD. First preliminary results have been presented at this conference [27].
} 


\section{References}

[1] R. Alkofer and L. von Smekal. Phys. Rept. 353, 281 (2001), [hep-ph/ 0007355$].$

[2] C. S. Fischer. J. Phys. G32, R253-R291 (2006), [hep-ph/ 0605173 ].

[3] C. D. Roberts and A. G. Williams. Prog. Part. Nucl. Phys. 33, 477 (1994), [hep-ph/ 9403224 ].

[4] C. D. Roberts, M. S. Bhagwat, S. V. Wright, and A. Holl. Eur. Phys. J. Special Topics 140, 53 (2007).

[5] L. von Smekal, R. Alkofer, and A. Hauck. Phys. Rev. Lett. 79, 3591-3594 (1997), [hep-ph/9705242]; Ann. Phys. 267, 1 (1998), [hep-ph/9707327].

[6] C. Lerche and L. von Smekal. Phys. Rev. D65, 125006 (2002), [hep-ph/ 0202194 ].

[7] D. Zwanziger. Phys. Rev. D65, 094039 (2002), [hep-th/ 0109224 ].

[8] D. Zwanziger. Phys. Rev. D69, 016002 (2004), [hep-ph/ 0303028 ].

[9] J. M. Pawlowski, D. F. Litim, S. Nedelko, and L. von Smekal. Phys. Rev. Lett. 93, 152002 (2004).

[10] J. M. Pawlowski. (2005), hep-th/0512261.

[11] R. Alkofer, C. S. Fischer, and F. J. Llanes-Estrada. Phys. Lett. B611, 279 (2005).

[12] C. S. Fischer and J. M. Pawlowski. Phys. Rev. D75, 025012 (2007), [hep-th/ 0609009 ].

[13] F. D. R. Bonnet, P. O. Bowman, D. B. Leinweber, A. G. Williams, and J. M. Zanotti. Phys. Rev. D64, 034501 (2001), [hep-lat/0101013]; P. O. Bowman, U. M. Heller, D. B. Leinweber, M. B. Parappilly, and A. G. Williams. Phys. Rev. D70, 034509 (2004), [hep-lat / 0402032 ].

[14] A. Sternbeck, E.-M. Ilgenfritz, M. Müller-Preussker, A. Schiller, and I. L. Bogolubsky. PoS (LAT2 006 ) 076 (2006), [hep-lat/ 0610053 ]; Braz. J. Phys. 37, 193 (2007), [hep-lat/0609043].

[15] C. S. Fischer, A. Maas, J. M. Pawlowski, and L. von Smekal. to appear in Ann. Phys. (2007), hep-ph/0701050.

[16] C. S. Fischer, R. Alkofer, A. Maas, J. M. Pawlowski, and L. von Smekal. Po.S (LATTICE $2007) 300$ (2007), [arXiv:0709.3205 [hep-lat]].

[17] A. Maas. Phys. Rev. D75, 116004 (2007), [arXiv:0704.0722 [hep-lat]].

[18] L. von Smekal. Presented at the international workshop Confinement: connecting the light-and heavy-quark domains, ECT*, Trento, Italy, March 2007.

[19] K. Langfeld. (2007), arXiv:0704.2635 [hep-lat].

[20] A. Sternbeck, E.-M. Ilgenfritz, M. Müller-Preussker, and A. Schiller. Phys. Rev. D72, 014507 (2005), [hep-lat/0506007].

[21] A. Sternbeck. The infrared behavior of lattice QCD Green's functions. PhD thesis, Humboldt-University Berlin, 2006. hep-lat/0609016.

[22] D. B. Leinweber, J. I. Skullerud, A. G. Williams, and C. Parrinello. Phys. Rev. D58, 031501 (1998)

[23] A. Cucchieri, A. Maas, and T. Mendes. Phys. Rev. D75, 076003 (2007), [hep-lat/ 0702022 ].

[24] A. Cucchieri and T. Mendes. PoS (LATTICE 2007) 297 (2007).

[25] I. Bogolubsky, V. Bornyakov, G. Burgio, E.-M. Ilgenfritz, V. Mitrjushkin, M. Müller-Preussker, and P. Schemel. PoS (LATTICE 2007 ) 318 (2007).

[26] A. Cucchieri, T. Mendes, O. Oliveira, and P. J. Silva. (2007), arXiv:0705.3367 [hep-lat]; arXiv:0710.0344 [hep-lat].

[27] A. Sternbeck et al. POS (LATTICE 2007 ) 256 (2007).

[28] I. Bogolubsky, E.-M. Ilgenfritz, M. Müller-Preussker, and A. Sternbeck. PoS (LATTICE $2007) 290$ (2007).

[29] L. von Smekal, D. Mehta, A. Sternbeck and A. G. Williams PoS (LATTICE 2007) 382 (2007). 